I hope we all stay physically and mentally healthy in these strange times.

K. Chan, Belfast, UK

https://doi.org/10.1038/s41415-020-2462-1

\section{Mental health training}

Sir, the awareness of the importance of our mental health within the dental profession has increased recently, which is amazing and vital but this alone is not enough. We know dentistry is a stressful career and a challenging university course, and our mental health can be significantly affected. Also, being healthcare professionals, we have a duty of care to patients who could have a range of mental health diagnoses or who could present in a crisis - for example having suicidal thoughts.

This leads to the question: why aren't we undertaking regular mental health and suicide training? Mental Health First Aid courses are available which should be compulsory to complete alongside physical first aid and medical emergencies training. ${ }^{1}$ Zero Suicide Alliance offers a free 20-minute training course on suicide awareness which highlights three key aspects: SEEing the signs of suicide; SAYing, speaking about suicide and whether someone has suicidal thoughts; and SIGNPOSTing people to the correct services, eg GP, Samaritans. ${ }^{2}$

If a colleague or patient is struggling with their mental health, we need to talk openly about suicide and whether they are having or have had suicidal thoughts. This can be difficult to do but is imperative to their safety and wellbeing. It does not 'put the idea into their head', but instead protects them and allows people to tell you how they feel. ${ }^{3}$

We need to urgently integrate this training into both the student curriculum and professional CPD cycles. Not only mental health training, but also thorough interactive equality and diversity training (eg by E\&D UK), ${ }^{4}$ since discrimination has a huge impact on mental health and is so prevalent in our society.

As we normalise talking about suicide, we should be using the correct terminology. For example, 'committed suicide' is a term that should be avoided as it implies it is a crime and adds to the stigma. Instead we can say 'died by suicide' or 'took their own life.'

We must also question: why is there a barrier to dental professionals seeking help for their mental health? More attention is needed on mental health within the profession to save lives. If you need someone to talk to or are experiencing suicidal thoughts, call Samaritans on 116 123, who are available 24/7.

\section{K. Benson, Newcastle, UK}

\section{References}

1. MHFA England. Mental health training online and face to face. 2020. Available at: https://mhfaengland.org/ (accessed October 2020).

2. Zero Suicide Alliance. ZSA Training. 2020. Available at: https://www.zerosuicidealliance.com/training (accessed October 2020).

3. Samaritans. Supporting someone with suicidal thoughts. 2020. Available at: https://www.samaritans.org/ how-we-can-help/if-youre-worried-about-someoneelse/supporting-someone-suicidal-thoughts/ (accessed October 2020).

4. Equality and Diversity UK. Equality and Diversity: Training. Resources. Network. 2020. Available at https://www. equalityanddiversity.co.uk/ (accessed October 2020).

5. Samaritans. How to talk about suicide safely online. 2020. Available at: https://www.samaritans.org/aboutsamaritans/research-policy/internet-suicide/how-talkabout-suicide-safely-online/ (accessed October 2020).

https://doi.org/10.1038/s41415-020-2463-0

\section{Medical emergencies}

\section{Three things}

Sir, I write further to an article published in the $B D J$ on 25 September entitled Management of odontogenic infections and sepsis: an update. ${ }^{1}$

Firstly, while recommending incision placement, the authors mention making the same on healthy skin or mucosa. It would be important to include here that skin incisions must be made as aesthetically as possible, keeping them parallel to (or in) existing skin tension lines. ${ }^{2}$ This would not only serve the purpose of the procedure but also enhance cosmetic outcomes.

The dimensions of the incision must allow for adequate access of the haemostat. An adequately sized incision enhances irrigation and avoids excessive build-up of pressure within the abscess cavity. ${ }^{2}$

Secondly, the authors recommend opening the haemostat 'at the depth of penetration'; a more specific way to ascertain this is to insert the haemostat till the resistance of healthy tissue is felt. ${ }^{2}$ Apart from a microbiological swab, a syringe can also be utilised to obtain a sample of the drainage. This should ideally be sent for culture sensitivity testing (CST). The article describes dentists being guardians against antibiotic resistance, especially due to the fallout from the pandemic. This makes recommending CST even more important in cases where the abscess has clinically worsened in follow up appointments and antibiotic treatment is necessitated.
Thirdly, the authors have also mentioned wrong antibiotic choice and/or dosage as a cause for treatment failure. Utilising CST would help to circumvent this and move away from protracted empirical regimens and their associated issues.

Finally, the authors make the correct suggestion of never closing a haemostat while it is inside the wound. It would be informative for readers to know that the reason behind this is to avoid damaging any vital structures in the vicinity. Knowing the reason and its gravity would serve to underscore such a suggestion.

\section{Sahni, New Delhi, India}

\section{References}

1. Jevon P, Abdelrahman A, Pigadas N. Management of odontogenic infections and sepsis: an update. Br Dent J 2020; 229: 363-370.

2. Fitch M T, Manthey D E, McGinnis H D, Nicks B A, Pariyadath M. Videos in clinical medicine. Abscess incision and drainage. N Engl J Med 2007; 357: e20.

https://doi.org/10.1038/s41415-020-2464-z

\section{Sepsis and COPD}

Sir, I write further to the excellent poster: Medical emergencies in the dental practice. ${ }^{1}$

With medical emergencies the risk of mortality multiplies with co-morbidities, especially so in managing sepsis, when organ dysfunction follows the deregulated response to infection. ${ }^{2}$ Undoubtedly in patients with both sepsis and COPD (chronic obstructive pulmonary disease) time management is critical.

Such patients demonstrate greater risks of acute exacerbation, pneumonia and mortality compared to those with one condition. ${ }^{3}$ While a single Red Flag Sign triggers blue light transfer, one sign: Needs oxygen to keep $\mathrm{Sp} \mathrm{O}_{2}$ 92\% (88\% in COPD) is noteworthy when managing the patient at risk of sepsis, but who has COPD. Discussion with the authors revealed the following:

1. The Sepsis Trust Screening Tool follows

NICE Guideline NG51 (1.4.2): high sepsis risk follows the need to maintain oxygen saturations more than $92 \%$ or more than $88 \%$ where COPD is known. ${ }^{4}$ This guideline is followed for medical but not dental practices, where $\mathrm{SpO}_{2}$ is to be kept at specific saturation levels ${ }^{1}$

2. With sepsis and COPD, pragmatic rather than dogmatic approaches are vital, the oxygen dissociation curve shifts left, partial oxygen pressure decreases and haemoglobin's oxygen binding increases. 